\title{
Análisis de seguridad y productividad del suministro de energía eléctrica en el sistema eléctrico de Nicaragua en el periodo comprendido desde el año 2010 hasta el 2018
}

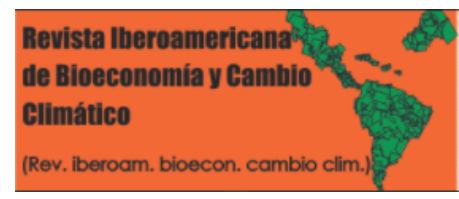

\section{Analysis of safety and productivity of power supply in the electrical system of Nicaragua in the period from 2010 to 2018}

\section{Blanco-Orozco, Napoleón; Zúniga-González, Carlos Alberto; Torres, Jaime; Arce-Díaz, Eduardo; Martínez-Andrade, Erick; Dios- Palomares, Rafaela; Quiros, Olman; Colon-García, Adelfa Patricia; Hernández-Rueda, Mauricio; Editor Academico Prof. Dr. Angel Sol Sanchez}

\author{
(iD) Napoleón Blanco-Orozco \\ napoleon.blanco@usr.uni.edu.ni \\ UNI, Nicaragua \\ Carlos Alberto Zúniga-González \\ czuniga@ct.unanleon.edu.ni \\ UNAN-León, Nicaragua \\ Jaime Torres \\ Ingeniero eléctrico, Nicaragua \\ Eduardo Arce-Díaz \\ earced@yahoo.com.mx \\ Kansas State University, United States \\ Erick Martínez-Andrade \\ UNAN-León, Nicaragua \\ Rafaela Dios-Palomares \\ ma1dipar@uco.es \\ Universidad de Córdoba, Spain \\ Olman Quiros \\ CURLA UNAH, Honduras \\ iD Adelfa Patricia Colon-García \\ CIEDA UCR, Costa Rica \\ (iD) Mauricio Hernández-Rueda \\ UNAN LEON, Nicaragua \\ Editor Academico Prof. Dr. Angel Sol Sanchez \\ Colegio de postgraduados, Mexico
}

\author{
Revista Iberoamericana de Bioeconomía y Cambio \\ Climático \\ Universidad Nacional Autónoma de Nicaragua, León, Nicaragua \\ ISSN-e: 2410-7980 \\ Periodicidad: Semestral \\ vol. 1, núm. 2, 2015 \\ czuniga@ct.unanleon.edu.ni
}

Recepción: 06 Junio 2015

Aprobación: 01 Octubre 2015
Resumen: El propósito de este estudio fue el de estudiar la seguridad y productividad del suministro de energía eléctrica en el sistema eléctrico de Nicaragua en el periodo desde el año 2010 hasta el 2018 utilizando el Indicador Stirling, indicador margen de reserva, indicador pérdida del mayor generador, el indicador de concentración de mercado y herramienta metodológica de análisis envolvente de datos (DEA) y los índices de Malmquist. Se estudió el comportamiento del sector energético de Nicaragua, en el periodo 2005 - 2013. Como resultado del indicador Stirling se obtuvo, que Nicaragua en estos momentos cuenta con seguridad en el sistema eléctrico, Del indicador margen de reserva se obtuvo como resultado, que el nivel de seguridad desde el punto de evaluación de este indicador el sistema cuenta con la suficiente seguridad como para no incurrir en afectaciones a los consumidores finales, Como resultado del indicador pérdida del mayor generador (LU), concluye en que se posee un sistema seguro. Por otra parte, El índice de concentración de mercado mostro que Nicaragua no posee un mercado energético competitivo. En la evaluación de productividad del uso de recursos energéticos se encontró que solo el uso de biomasa presenta un ritmo promedio anual de crecimiento de productividad.

Jel Classiffication: C:5; O:3; Q:43;

Palabras clave: Índices de Malmquist, Productividad, Energía eléctrica.

Abstract: The purpose of this study was to study the safety and productivity of power supply in the electrical system of Nicaragua in the period from 2010 to 2018 using the Stirling pointer, reserve margin, loss indicator largest generator, the market concentration indicator and methodological data envelopment analysis tool (DEA) and Malmquist indices. The behavior of the energy sector in Nicaragua, in the period studied 2005 - 2013. As result, an indicator from Stirling index ware obtained, right now Nicaragua has security in the electrical system. The reserve margin indicator was obtained as a result, of the safety level from the point of evaluation of this indicator the 
URL: http://portal.amelica.org/ameli/journal/394/3941749005/ DOI: https://doi.org/10.5377/ribcc.v1i2.2476

Autor de correspondencia: napoleon.blanco@usr.uni.edu.ni system has enough security to avoid incurring damages to final consumers, as a result of the loss of the largest generator indicator (LU), concludes that it has a secure system. Moreover, the rate of market concentration showed that Nicaragua does not have a competitive energy market. In assessing productivity use of energy resources, it ware found that, only the use of biomass has an average annual rate of productivity growth.

Jel Classiffication: C:5; O:3; Q:43;

Keywords: Malmquist Index, Productivity, Electrical Energy.

\section{INTRODUCCION}

El propósito de este estudio fue el de estudiar la seguridad y productividad del suministro de energía eléctrica en el sistema eléctrico de Nicaragua en el periodo desde el año 2005 hasta el 2014 utilizando Indicador Stirling, indicador margen de reserva, indicador pérdida del mayor generador, el indicador de concentración de mercado y herramienta metodológica de análisis envolvente de datos (DEA) y los índices de Malmquist.

Según datos del Banco Mundial el incremento poblacional porcentual en el mundo desde 2010 hasta 2014 ha sido del 1.5\% BM, (2015), mientras que la demanda de energía a escala mundial crecerá un 2,4\% anual (CIEMAT, 2015), esto muestra que el crecimiento poblacional y el consumo de energía, no tienen un crecimiento proporcional. De la relación del crecimiento poblacional mundial con respecto al aumento del consumo de energía eléctrica, es posible prever proyecciones del consumo de energía para los próximos años con crecimiento vertical de la demanda de energía eléctrica.

Por otra parte, la importancia que tiene el suministro de energía eléctrica se refleja en el hecho de que en la actualidad casi toda actividad productiva requiere de electricidad, desde la producción de equipos y maquinarias, pasando por el vestuario y el calzado, la producción y empaque de los alimentos, hasta el bombeo de agua potable a nuestras viviendas. Todo lo mencionado anteriormente hace referencia a una necesidad del suministro de energía eléctrica, por tanto el desarrollo de un país depende del desarrollo del sistema eléctrico de cada nación.

En este momento Nicaragua se encuentra en pleno desarrollo del Sistema Interconectado Nacional (SIN), introduciendo nuevas plantas generadoras, nuevas subestaciones, líneas de transmisión, ampliación de las redes de distribución para llegar a abastecer los nuevos asentamientos y urbanizaciones que cada día son más (MEM, 2013). Ante la necesidad de generar energía eléctrica para abastecer la demanda se contrapone una gran cantidad de factores como: sociales, políticos, y los de mayor peso, los económicos, que hacen que los costos de generación de energía eléctrica, su transporte y la distribución hasta los consumidores finales sean fluctuantes y variables en el tiempo.

La Matriz Energética de Nicaragua (MEN) se encuentra vulnerable a las constantes variaciones del precio de los combustibles, porque aún se encuentra en un 56\% dependiente de las plantas térmicas (MEM, 2013). Además hay que considerar que las plantas generadoras tienen afectaciones en su operación que repercuten en la confiabilidad del sistema eléctrico de potencia como los cambios en el clima que afectan a las generadoras con fuentes renovables como es el caso de las Hidroeléctricas que generan a media capacidad en tiempos de sequía cuando el caudal de los embalses disminuyen, y también es el caso de las eólicas que en algún momento del día pierden gran parte de su capacidad de generación debido a escasez o exceso de viento. Todo esto genera para los gobiernos preocupación para lograr el aseguramiento del suministro de energía y anticiparse

\section{Notas DE AUTOR}


a las afectaciones como las del clima que es un factor externo e incontrolable con potencial de ocasionar insuficiencia de fuentes de energía eléctrica en distintos meses del año.

Lo mencionado con anterioridad es motivo para tener pleno interés en la seguridad del suministro energético, aplicando indicadores cuantitativos y cualitativos para determinar así la capacidad del SIN de responder a la demanda de energía eléctrica y la robustez del SIN ante cualquier eventualidad desfavorable que se presente.

Este artículo aborda la seguridad de suministro y la productividad del uso de recursos energéticos para la generación de energía eléctrica dejando claro que lo evaluado es la seguridad del sistema eléctrico y no la confiabilidad, porque cuando se habla de seguridad incluye la diferencia entre seguridad y confiabilidad radica principalmente en que la confiabilidad se encarga de evaluar el comportamiento del sistema en el corto plazo atendiendo principalmente aspectos medioambientales, afectaciones y estado de las estructuras de líneas de trasmisión, robustez de las redes de distribución, etc. mientras que la seguridad del suministro es un análisis mucho más completo en el que se encarga de medir la confiablidad, robustez, diversidad de todo el sistema eléctrico nacional. (Ventosa, 2010). El artículo se divide en cinco partes comprendiendo la introducción al tema de estudio, el sustento de la literatura en marco teórico, la metodología, los resultados y su análisis y las conclusiones.

\section{MARCO TEORICO}

Uno de los objetivos de los procesos de independencia y liberalización de los mercados de energía es alcanzar un suministro energético seguro. De forma general, podemos definir seguridad de suministro energético como la seguridad de obtener energía (eléctrica) a un nivel de precios que los consumidores estén dispuestos a pagar (Retamales, 2005 citando a Helm, 2002).

De manera que, existen una cantidad considerable de indicadores que evalúan la seguridad del suministro utilizados a nivel mundial para determinar la sostenibilidad del sistema energético de un país, divididos en dos tipos: los cualitativos y los cuantitativos. Y dentro de los más usados están:

El índice de seguridad de suministro de la Unión Europea (SoS) ec.1 , ec 2, ec3 que parte de una relación entre el suministro y la demanda del sistema, asignando un determinado peso a cada uno de los criterios que evalúa el modelo. Este indicador define una política de seguridad en general, que se compone de las medidas adoptadas para reducir los riesgos de perturbaciones en el suministro por debajo de un cierto nivel tolerable (Fergal et al., 2007).

El índice de seguridad de suministro de la Unión Europea establece que la seguridad de suministro se encuentra dada por:

$$
\operatorname{SoS}_{\text {index }}=\left(\frac{2}{3}\right)\left(\frac{S}{D_{\text {index }}}\right)+\frac{1}{3} \mathrm{CC}_{\text {index }}
$$

En donde la relación entre la demanda y el suministro y el índice de capacidad de crisis se encuentran dados por lo que se sigue:

$$
\begin{gathered}
\frac{S}{D}_{\text {index }}=(3.3) \text { valordedemanda }+(0.7) \text { valordeof erta } \\
\mathrm{CC}_{\mathrm{index}}=\frac{\mathrm{MA}}{\mathrm{RA}} \times 100\left(\mathrm{sLRA}, \leq, \mathrm{CC}_{\mathrm{index}}\right)=100
\end{gathered}
$$


A su vez, el índice de capacidad de crisis, establece una relación entre la evaluación del riesgo y la posibilidad de minimización del mismo a partir de un checklist de los diversos elementos establecidos para la evaluación del riesgo del sistema.

Otro indicador utilizado es el indicador Stirling - (IS) grafico 1 y que puede definirse como una propiedad irreducible de un sistema o como un atributo de un sistema que puede dividirse en categorías (Stirling, 2003). El indicador Stirling se centra en evaluar la diversidad de un sistema considerando los elementos que definen a este indicador: la variedad, el equilibrio y la disparidad de las fuentes de energía.

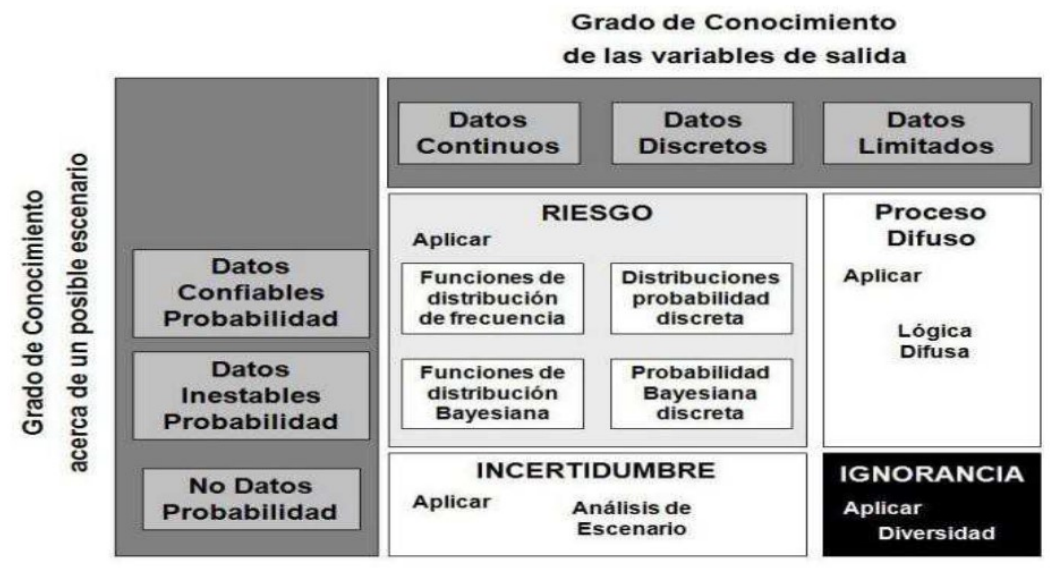

GRAFICO 1.

Matriz de análisis del sistema del indicador Stirling. Fuente: J. Molina, V. Martínez, H. Rudnick, indicadores de seguridad energética: Aplicación al sector energético de Chile. Indicadores de seguridad energética: Aplicación al sector energético de Chile. J. Molina, V. Martínez, H. Rudnick,

El Indicador Stirling define la variedad como el número de categorías en las que los elementos del sistema se reparten, todo con igualdad de condiciones, cuanto mayor sea la variedad mayor será la diversidad; el equilibrio como al patrón en la distribución de la cantidad de un elemento específico a través las categorías pertinentes. Para un sistema, entre más iguales sean las fracciones mayor será la diversidad. Y la disparidad como el grado de disimilitud entre dos determinados objetos o tipos, el sistema que contiene las opciones más dispares será considerado el más diverso.

El indicador de Stirling (ec 4) es el resultado de la comprensión de índices de variedad (Shannon - Weiner), balance (Herfindahl), y disparidad (Weitzman), agrupados en un índice multicriterio aplicado a muchos campos de acción (política, tecnología) y distintas ciencias (economía, la biología), que incluye un estudio completo de los tres componentes de la diversidad. El índice de Stirling planteado para la evaluación de la diversidad se denota con la letra M:

$$
M=\sum d_{\mathrm{ij} *} P_{i} P_{j}
$$

Dónde:

$\mathrm{M}=\sum \operatorname{dij} \times \mathrm{pi} \times \mathrm{pj}$

ij $(i \neq j)$

dij = Distancia euclidiana entre la opción i y la opción j.

pi $=$ Es la proporción de la opción i pj $=$ La representación de opción $\mathrm{j}$

Otro indicador es el indicador de sustentabilidad energética - (ISE) donde se define a la sustentabilidad energética como el equilibrio entre tres dimensiones principales: la seguridad energética, la equidad social, y la 
mitigación del impacto ambiental. El desarrollo de sistemas de energía, estables, accesibles y ambientalmente aceptables es lo que se pretende alcanzar cuando se habla de sustentabilidad energética (Molina et al., 2009).

Por su parte, el indicador de sustentabilidad energéticas (ISE) grafico 2 es utilizado para establecer el grado de sustentabilidad energética basado en autonomía energética, robustez ante cambios externos, productividad energética, cobertura del sistema eléctrico y de necesidades básicas energéticas, impacto ambiental del energético, uso de fuentes renovables y estimación del uso de dichas fuentes. Las variables mencionadas anteriormente establecen una alta sustentabilidad de la autarquía con una baja participación de las importaciones de la oferta energética y la robustez con una baja contribución al PIB de las exportaciones energética. Para la obtención del ISE en general, es necesario conocer antes todos lo sub indicadores descritos en la tabla siguiente, detallando en cada uno la relación con la sustentabilidad y a que objetivos obedecen.

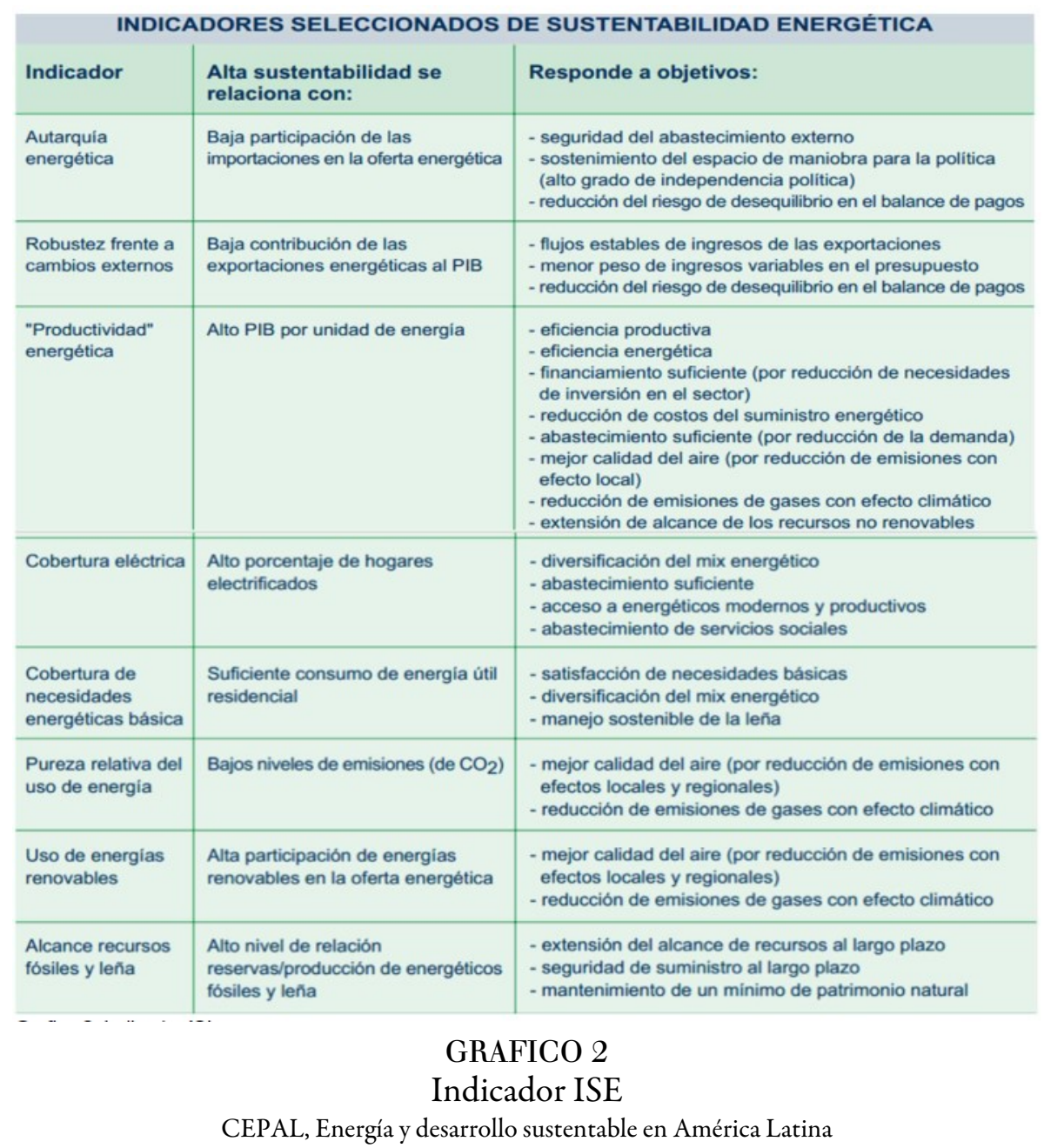

Otro indicador es el indicador de robustez del sistema eléctrico - (RR) y se expresa a través de la Rosa de Robustez (RR) que consiste en un análisis gráfico que considera variables económicas y técnicas, en lo que se refiere a la seguridad de suministro. Los parámetros que pretende evaluar son esencialmente la diversificación de la matriz energética, la instalación de fuentes autóctonas, el incremento de energía firme y la generación de mayor valor agregado al país (Molina et al., 2009).

El indicador $\mathrm{RR}$ valora el costo del suministro según las fuentes empleadas en la producción y la seguridad de su obtención; en síntesis las incertidumbres que sobresalen en esta metodología son: variaciones de precio y disponibilidad para obtener las fuentes de generación (por ejemplo petróleo), disponibilidad y precio de otros energéticos, y la aleatoriedad de los recursos renovables propios. 
Otro indicador es indicador pérdida esperada de carga - (LOLE) en el cual se evalúa la seguridad del suministro en base en valores esperados de pérdida de carga, es decir cuántas horas anuales en energía no se podrán suministrar al sistema. Dicha metodología se conoce como Loss of Load Expectation - LOLE. En general, el máximo valor encontrado de LOLE se utiliza para configurar el sistema eléctrico, tal que, el riesgo de no poder satisfacer la demanda sea aceptable. El indicador propone evaluar la seguridad del suministro en base a la capacidad del sistema eléctrico de enfrentar el crecimiento de la demanda. Esto se traduce en la cantidad mínima de capacidad instalada efectiva que requiere la MEN para garantizar el suministro a los consumidores finales.

Otro indicador es el indicador de margen de reserva - (reserve margin - RM) y se define como el excedente de la capacidad de generación disponible para satisfacer la demanda máxima anual. Este indicador establece un valor cuantitativo de cuanto sobrepasa la capacidad instalada a la demanda anual nacional. Los requerimientos necesarios para el cálculo de este indicador son: demanda máxima anual de potencia y la capacidad instalada efectiva anualmente.

Otro indicador es el indicador de pérdida del mayor generador - (largest unit -

LU) que considera la posible indisponibilidad del mayor generador. Este indicador muestra la situación del sistema eléctrico nacional en caso de que se presentara la indisponibilidad de la planta generadora de mayor capacidad presente en la Matriz Energética de Nicaragua (MEN), las afectaciones que se derivan de esta contingencia y como afecta esto principalmente a la seguridad del suministro de energía eléctrica. Este indicador es como una continuación del indicador margen de reserva y por ende para su cálculo es necesario conocer antes el RM, luego de esto se necesita conocer la planta de generación más grande presente en cada año de estudio.

Otro índice es índice de concentración de mercado - (ICM) conocido como Índice de Herfindahl e Hirschman, (IHH) que es una medida de la concentración de un mercado y/o en este caso de la MEN, desde el punto de vista inverso es la medida de la falta de competencia de un sistema. A más alto el índice, más concentrado, menos competitivo, el mercado. El índice se calcula elevando el cuadrado el porcentaje de aporte de cada una de las fuentes primarias de generación de energía eléctrica presentes en la MEN, y haciendo una sumatoria de cada resultado para obtener el valor final del índice. Los resultados van desde cerca de 0 (cero, competencia perfecta) hasta 10000 (diez mil, control monopólico).

Por otro lado, la medición de la eficiencia de una empresa consiste en medir su desempeño con respecto a un óptimo. En este sentido, el método del análisis envolvente de datos (DEA), permite estudiar la eficiencia de una unidad de producción con respecto al desempeño de otras unidades de producción similares, a partir de la construcción de la frontera eficiente mediante aproximaciones no paramétricas (Quindós et al., 2003).

La frontera eficiente se define por la actuación de las mejores empresas observadas, que servirá como referencia para medir la eficiencia relativa de cada firma al compararse con dicha frontera. En un análisis DEA se realizan dos procesos simultáneamente mediante el uso de algoritmos de programación lineal: la obtención de la frontera eficiente y la estimación de la ineficiencia. La obtención de la frontera eficiente se calcula maximizando el output dado el nivel de inputs si se utiliza output orientado y minimizando el input dado el nivel de outputs si se utiliza orientación input.

La estimación de la ineficiencia depende de la orientación utilizada y se calcula como la distancia a la frontera de cada empresa evaluada, comparándose cada empresa con otra tecnológicamente similar. Además, la eficiencia de una unidad de generación se calcula empleando variables de entradas y salidas y empleando la técnica de programación lineal el análisis DEA que permite a partir de la mejor practica observada considerada como frontera eficiente, evaluar la eficiencia de una unidad de producción cualquiera (Pedraja y Jiménez, 1994).

Por otra parte, el método DEA se trata de una herramienta de análisis económico cuantitativo válida para estudiar el desempeño de unidades productivas, sectores y países y que posee la ventaja de facilitar un tratamiento multidimensional, tanto del lado desde el espacio de los insumos o factores como del de los 
productos con que se trabaje. A partir de esta metodología es posible precisar la frontera tecnológica basada en unidades productivas que, por sus buenos resultados, son consideradas como aquellas que realizan las mejores prácticas productivas en relación a las otras unidades. De esta forma, se establece una frontera de referencia a través de la cual es posible definir medidas de eficiencia productiva, sobre la base del cálculo de las distancias que median entre cada unidad productiva y dicha frontera (Schuschny, 2007).

Por lo que, DEA consiste en categorizar el desempeño de las unidades productivas que participan del estudio, mediante la identificación de unidades pares (eficientes) a partir de las cuales se construyen otras unidades (virtuales) que resultan comparables y, gracias a las cuales se calculan los indicadores de eficiencia y sus cambios a lo largo del tiempo. De esta forma, el análisis nos permite identificar aquellas unidades de producción que mejor desempeño han tenido en términos de su eficiencia en el uso de input para optimizar el output.

Por otro lado, el uso de índices Malmquist de productividad tiene como ventaja el permitir descomponer el cambio de la productividad en sus elementos determinantes a lo largo del tiempo y para detectar adopción de nuevas tecnológicas involucradas en el sistema productivo. Los índices Malmquist representan el crecimiento de la productividad total de los factores (TFP) de una unidad de decisión (DMU) y refleja el progreso en eficiencia en consonancia con el progreso de la frontera tecnológica en el tiempo; es decir, las unidades inicialmente eficientes, incrementan su productividad en el tiempo, bajo un marco de múltiples insumos y productos (Ferro y Romero, 2011).

Así mismo, mediante el empleo de DEA se cuantifican los cambios a lo largo del tiempo, recurriendo al uso de los índices de Malmquist que nos permiten discriminar entre los cambios en la eficiencia técnica (o sea cambios en la distancia a la frontera, lo que se denomina como convergencia o "catching-up") y los cambios tecnológicos (que se manifiestan como desplazamientos de la propia frontera), así como determinar los cambios en la productividad total de los factores. Por otro lado, este instrumento de análisis nos permite identificar los cambios de eficiencia pura respecto de los cambios debido a la modificación de la escala de producción.

El índice de Malmquist, al estar basado en el método DEA presenta ventajas como el hecho de que permite trabajar con múltiples insumos y productos, medidos en diferentes sistemas de unidades y al contrario de los métodos de naturaleza econométrica que emplean una aproximación a la frontera de producción estocástica, la estimación del índice de Malmquist no requiere la asunción de pleno empleo de los factores productivos. Además, el índice de Malmquist no impone el uso de formas funcionales explícitas para la tecnología y se puede descomponer en cambio técnico y cambio en la eficiencia y, por lo tanto, aporta información sobre los efectos relativos de estos factores (Flores et al., 2014) y (Blanco et. al. 2014).

Adicionalmente, el avance en la productividad total de los factores (PTF) nos indica una mejoría en la tecnología y en la organización de la producción. El enfoque de la medición de la productividad a través de números índices se define como la relación de un índice de output (Q) respecto a un índice de inputs $(\mathrm{P})$.

El índice de Malmquist (ec 5, ec 6, ) es definido usando la función distancia. Una función distancia input caracteriza la tecnología de la producción buscando proporciones mínimas del vector input, dado un vector output; una función de distancia output considera maximizar proporcionalmente y expandir el vector output, dado un vector input.

Una tecnología de la producción puede ser definida usando el output:

$$
P(X)=(y: \text { Xpuedeproduciry })
$$

La función de distancia output es definida en el output, $\mathrm{P}(\mathrm{x})$, como:

$$
d_{0}(x, y)=\min \left[\delta:, \frac{\gamma}{\delta}, \varepsilon \mathrm{P},(X)\right]
$$


El índice de Malmquist DEA es un método para estimar funciones fronteras (maximización, minimización, etc.,) utilizando el análisis de datos envolventes estas distancias son: a) La frontera DEA con tecnologías a rendimientos a escala constante del período previo, b) la frontera DEA a con tecnologías rendimientos de escala constante del período actual, c) la frontera DEA con tecnologías a rendimientos de escala constante del siguiente período, y d) la frontera DEA con tecnologías a escala de rendimientos decrecientes.

El modelo parte de considerar que hay datos en K inputs y M outputs de cada N Unidades de producción (UP), para la i-ésima UP que son representados por el vector $\mathrm{x} 1$ y y 1 , respectivamente. La matriz insumo $\mathrm{K} \mathrm{x}$ $\mathrm{N}, \mathrm{X}$, y la matriz producto $\mathrm{Mx} \mathrm{N}, \mathrm{Y}$, representa los datos de todas las unidades de producción. El propósito de DEA es construir una frontera envolvente no paramétrica sobre los datos señalados como los observados bajo frontera de producción.

La mejor manera de introducir DEA ec7 (PL 1) es por la vía de razones. Para cada unidad de producción a los productores les gustaría obtener la medida de razón de todos los productos sobre todos los insumos, tal como u' yi/v' xi, donde u es un $\mathrm{Mx} 1$ vector de pesos output y v es un vector de pesos inputs. Para seleccionar los pesos óptimos especificamos un problema de programación matemática:

$$
\left|\begin{array}{c}
\operatorname{Max} u v\left(\frac{u^{\prime} y_{i}^{\prime}}{u^{\prime} x^{\prime}}\right) \\
s . a\left(\frac{u^{\prime} y_{i}^{\prime}}{u^{\prime} x^{\prime}}\right) \\
\mathrm{u}, v \geq 0
\end{array}\right|
$$

Implica encontrar valores de u y v, tal que la medida de eficiencia de la i-ésima finca maximizada, sujeto a la restricción que todas las medidas de eficiencias deben ser menos o igual que uno. Un problema con la formulación de esta particular razón es que tiene infinito número de soluciones (Coelli, 1996), para evitar esto se puede plantear la restricción v' $\mathrm{x}_{\mathrm{i}}=1$, la cual provee PL 2:

$$
\left|\begin{array}{c}
\max _{u v}\left(\mu y_{i}\right) \\
s . a v x_{i} \leq 0 j=1,2, \ldots \ldots \ldots \ldots . . . N \\
\mu, v \geq 0
\end{array}\right|
$$

Este procedimiento matemático (PL 3) refleja la transformación conocida como el multiplicador de la forma del problema de programación lineal. Utilizando la dualidad en la programación lineal uno puede derivar una forma envolvente equivalente de este problema (Coelli: 1996).

$$
\left|\begin{array}{cc}
\min _{\mathrm{xy}} & \theta \\
\text { s. } a-y_{i}+\lambda \geq 0 & \\
\theta x_{i}-x \geq 0 & \lambda \geq 0
\end{array}\right|
$$

El problema de programación lineal deber ser resuelto $\mathrm{N}$ veces, una vez para cada unidad de producción en la muestra. Un valor de $\theta$ es entonces obtenido para unidad de producción de energía o planta generadora.

El índice de cambio de productividad de Malmquist se expresa como: PL 4 


$$
m\left(y_{t+1}, x_{\mathrm{t}+1}, Y_{t}, X_{t}\right)=\sqrt{\left[\frac{d_{0}^{t}\left(, X_{\mathrm{t}+1}, Y_{\mathrm{t}+1}\right)}{d_{0}^{t}\left(, X_{t}, Y_{t}\right)}, \times, \frac{d_{0}^{t+1}\left(, X_{\mathrm{t}+1}, Y_{\mathrm{t}+1}\right)}{d_{0}^{t}\left(, X_{t}, Y_{t}\right)}\right]}
$$

Esto representa la productividad de la producción en el punto $(x t+1, y t+1)$ relativo al punto de producción $(\mathrm{xt}, \mathrm{yt})$. Un valor mayor que uno indicará crecimiento del TFP del período $\mathrm{t}$ para el período $\mathrm{t}+1$. Un valor mayor de uno indicara un crecimiento positivo de PTF del período $t$ al periodo $t+1$.

Para calcular la programación lineal debemos calcular las cuatro funciones de distancia de los componentes, que se involucran los problemas de la programación lineal (P L), se asume REC de forma tal que los planteamientos de programación serán: $\mathrm{Pl}$ 5, Pl 6, Pl 7 y Pl 8 :

$$
\begin{aligned}
& \left|\begin{array}{cc}
d_{0}^{t}\left(\left(, X_{t}, Y_{t}\right)^{-1}\right)=\max _{\phi \lambda} \theta & \\
\text { s.a } a-\phi Y_{i t}+\gamma \lambda \geq 0 & \\
X_{\mathrm{it}}-X_{t} \lambda \geq 0 & \lambda \geq 0
\end{array}\right| \\
& d_{0}^{t}\left(\left(, X_{t}, Y_{t}\right)^{-1}\right)=\max _{\phi \lambda} \theta \\
& \text { s. } a-\phi Y_{i t+1}+\gamma \lambda \geq 0 \\
& X_{\text {it }}-X_{t} \lambda \geq 0 \quad \lambda \geq 0 \\
& d_{0}^{t}\left(\left(, X_{\mathrm{t}+1}, Y_{t+1}\right)^{-1}\right)=\max _{\phi \lambda} \theta \\
& \text { s.a }-\phi Y_{i t}+\gamma \lambda \geq 0 \\
& X_{\text {it }}-X_{t} \lambda \geq 0 \quad \lambda \geq 0 \\
& d_{0}^{t}\left(\left(, X_{t}, Y_{t}\right)^{-1}\right)=\max _{\phi \lambda} \theta \\
& \text { s. } a-\phi Y_{i t}+\gamma_{t} \lambda \geq 0 \\
& \lambda \geq 0
\end{aligned}
$$

Los puntos de producción son comparados con tecnologías de diferentes tipos de períodos, el parámetro $\theta$ no necesita ser $\geq 1$, como debe ser cuando calculamos la eficiencia de Farell. Los $\theta$ y $\lambda$ pueden tomar valores diferentes cuatros planteamiento de la programación lineal (PL). Además, los cuatro problemas de programación lineal deben ser calculados para cada unidad de producción en la muestra (UP).

\section{Metodología}

\section{Evaluación de seguridad de suministro de energía eléctrica del periodo 2007 al 2014.}

Para la evaluación de la seguridad de suministro en este estudio se consideraron índices de carácter cuantitativo y solamente uno es cualitativo, cada uno fue desagregado en sus variables de entrada/salida y fueron seleccionados de acuerdo a la información disponible, y de esta manera se determinó su aplicación al 
sistema energético de Nicaragua, estudiando detalladamente los requerimientos de cada indicador para su correcta aplicación.

Se presentan las justificaciones debidas y se determina cuáles son los indicadores de seguridad del suministro posibles de aplicar al sector energético de Nicaragua, considerando la variedad de indicadores que se puedan aplicar a la Matriz Energetica de Nicaragua (MEN) desde muchas perspectivas.

\section{Evaluación de productividad del uso de recursos energéticos en la generación de energía eléctrica}

Para el análisis de productividad se consideran como objeto de estudio los recursos energéticos empleados para la generación de energía eléctrica excepto el recurso eólico. El análisis de productividad trata la relación existente entre el (o los) productos y los insumos y su medición parte de la cuantificación de la producción obtenida y los insumos utilizados en el proceso de transformación productiva.

Para la evaluación de la productividad del uso de energéticos en la generación de energía eléctrica en el periodo 2005 al 2014 se empleó el método de análisis de datos envolvente (Data Envelopment Analysis, DEA). Así mismo, se utilizaron datos de panel para calcular cambios en los índices de productividad total de factores (PTF); cambio tecnológico; cambio de eficiencia técnica y cambio de escala de eficiencia. Para el cálculo, se hizo uso del programa de simulación DEAP 2.1 que contiene los algoritmos del proceso metodológico del método Malmquist; Se empleó la medida de Output-Orientado para comparar la productividad del uso de cada energético.

\section{Panel de datos, DEA}

Los datos empleados en el análisis son la entrada y salida de los procesos de generación de energía eléctrica de tipos de centrales que funcionan actualmente en Nicaragua y que están conectadas al sistema interconectado nacional. Los datos analizados en esta sección fueron obtenidos de los sitios web de instituciones del estado de Nicaragua como el Instituto Nicaragüense de la Energía (INE), el Despacho Nacional de Carga (CNDC) y del Ministerio de energías y Minas de Nicaragua (MEM). Los datos analizados fueron:

Salida: Se utilizó generación neta GW-HR, durante el periodo de estudio.

Entradas: Capacidad de potencia instalada en MW y el insumo para la generación, durante el periodo de estudio.

Cobertura: Se utilizó una serie de tiempo entre el 2005 y 2014.

\section{RESULTADOS Y DiscusióN}

Con respecto al índice de seguridad de suministro de la Unión Europea (SoS), este índice de seguridad de suministro no puede ser aplicable al sistema energético de Nicaragua porque el cálculo del índice de capacidad de crisis (CCindex) requiere de una lista de chequeo en donde se establezcan los valores de cada una de las fuentes primarias de energía, considerando diversos aspectos económicos y técnicos y asignándoles un valor a la evaluación del riesgo (RA) y la mitigación del riesgo (MA). El principal inconveniente de este índice, es que no se cuenta con la información que requiere el CC index para su aplicación a la MEN. No se cuenta con la información que requiere el índice SoS, colocando el indicador como inaplicable en este momento a sector energético de Nicaragua.

El indicador Stirling que propone evaluar la diversificación de un sistema de manera completa y la diversificación se convierte en un medio para conseguir evitar o minimizar el impacto de los riegos provocados por un incremento de la inseguridad energética. Los datos necesarios para la evaluación del índice Stirling 
de diversidad son accesibles, lo que coloca a este indicador en una situación aplicable al sector energético de Nicaragua.

En cuanto al indicador de sustentabilidad energética por la complejidad de los requerimientos y la obtención de los datos para la aplicación del indicador, se considera inaplicable el sector energético de Nicaragua, porque no se cuenta con la gran cantidad de información que el indicador requiere. Adicional a esto la aplicación de este indicador, ha sido realizada únicamente por organismos internacionales que se dedican el monitoreo de la todos los sectores que participan en la actividad económica de los países de América Latina y el Caribe, organismos como el CEPAL y el OLADE.

Con respecto al indicador de robustez del sistema eléctrico y Rosa de Robustez se buscaría realizar una evaluación de los planes de expansión de la MEN aplicado en años anteriores (2000 - 2012 incluyendo los modelos de expansión de la generación estudiados para el 2018, considerando multiplicidad de aspectos que consideran el desarrollo de una estrategia de diversificación segura y robusta. Es una metodología para la evaluación más completa, desafortunadamente no es posible aplicarla, porque no se encuentra disponible la información de la energía firme del país, esto limita la aplicación de este indicador porque de este dato dependen los primeros tres componentes del indicador, posicionando al indicador como inaplicable a la MEN.

En cuanto al indicador pérdida esperada de carga para la obtención de los datos requeridos para presentar conclusiones sobre el comportamiento de la demanda y oferta de energía eléctrica en Nicaragua es necesario contar con un intercambio de información con los miembros de la bloque de producción (generación) y la institución encargada del control y monitoreo de la demanda, es decir, se debe realizar un historial del comportamiento estadístico acerca de las contingencias y sobre la curva de demanda del SIN, considerando las ocasiones del año en el que la demanda pico o demanda máxima ha superado la capacidad efectiva de generación, o considerar, al menos una vez en diez años, es decir que el SIN presentará déficit o superávit al menos una vez en diez años. Debido a los requerimientos del indicador, es inaplicable, porque este requiere un periodo de tiempo mínimo de un año para monitorear el comportamiento del sistema eléctrico nacional, resultando de este monitoreo el total de ocasiones en las que la demanda de energía no pudo ser cubierta por la generación de la misma y esta información no está disponible este es el principal inconveniente que posee este que lo hace inaplicable para este estudio.

El indicador margen de reserva es sencillo de cálculo, y requiere de datos sencillos y estos datos se encuentran disponibles para la aplicación al sistema eléctrico de Nicaragua, por tanto este es aplicable al sistema eléctrico de Nicaragua.

El indicador pérdida del mayor generador que evalúa la seguridad del suministro al momento que la planta de mayor capacidad instalada no se encuentre disponible para la generación de energía necesita de una información accesible.

El índice de concentración de mercado es un índice fácil en su aplicación, con poca información necesaria, pero con gran variedad de usos y aplicaciones en las ciencias y la ingeniería, evaluando la competitividad de un mercado, aplicado principalmente en análisis de mercado, para evaluar la situación a la que se enfrentaría una nueva empresa al querer ingresar al mercado, a mayor el valor del índice, mayor es garantía de penetrar el mercado, debido a que hasta ese momento se encuentra muy concentrado (monopolio). Es aplicable a la MEN y su resultado y debidas conclusiones y recomendaciones serán evaluadas más adelante.

\section{Aplicación de los indicadores}

\section{Indicador Stirling}

En este momento contamos con cinco tipos de fuentes primarias para la generación de energía eléctrica, que son: Térmicas, Hidroeléctricas, Geotérmicas, Eólicas y Biomasa, de un total de 8 fuentes usadas principalmente para generación de energía eléctrica. Por lo tanto, se asigna el orden que poseen las fuentes de 
generación aleatoriamente para encontrar la distancia euclidiana, que representa la disparidad de las fuentes de Energía primaria. En la tabla 1 se muestra el valor que se le asigna a cada una de las fuentes, de manera que se le asigna un valor de 9 a las plantas térmicas por el hecho de ser las de mayor presencia en la Matriz energética, luego 7 a las plantas hidroeléctricas porque no se parece en nada a las plantas térmicas, en cuanto al energético o fuente primaria que utilizan, y esto permite decir que hay disparidad de 2 espacios euclidianos, y así sucesivamente con el resto de plantas.

TABLA 1.

Disparidad de plantas de generación hasta el 2014

\begin{tabular}{|c|c|c|}
\hline \multicolumn{3}{|c|}{$\begin{array}{l}\text { Disparidad de plantas de generación hasta el } \\
2014\end{array}$} \\
\hline Planta & Porción & Disparidad \\
\hline Térmica & 0.54 & 9 \\
\hline Hidroeléctrica & 0.105 & 7 \\
\hline Geotérmica & 0.076 & 5 \\
\hline Eólica & 0.16 & 3 \\
\hline Biomasa & 0.103 & 1 \\
\hline
\end{tabular}

Tabla 1.

La tabla 2 muestra el porcentaje de participación de cada fuente en la MEN actual 2014, dato que se requiere para la aplicación del indicador además del valor de disparidad de cada fuente asignado de manera aleatoria. En este caso el valor de disparidad fue asignado de acuerdo al porcentaje de participación que obedeció la Matriz energética en el periodo de tiempo evaluado en el estudio, entonces el valor asignado a cada fuente de generación de energía, se debe al porcentaje de participación de cada fuente en la MEN desde el año 2000 hasta el 2014.

TABLA 2.

Porcentaje de participación en la Matriz de Generación de cada planta hasta el 2014.

\begin{tabular}{|l|l|}
\hline \multicolumn{2}{|l|}{$\begin{array}{l}\text { Porcentaje de participación en } \\
\text { la Matriz de Generación de } \\
\text { cada planta }\end{array}$} \\
\hline Planta & $\begin{array}{l}\text { Porcentaje de } \\
\text { participación }\end{array}$ \\
\hline Térmica & 0.63528331 \\
\hline Hidroeléctrica & 0.12310052 \\
\hline Geotérmica & 0.10574539 \\
\hline Eólica & 0.11142163 \\
\hline Biomasa & 0.06901779 \\
\hline
\end{tabular}

Tabla 2.

Se realiza la combinatoria de cada una de las 5 fuentes primarias de generación de energía eléctrica existentes en Nicaragua, operación en la que se obtienen 10 resultados diferentes del argumento de la sumatoria (dij x pi x pj,). Una vez calculados todos los valores se suman para obtener el valor final que es el Índice de diversidad de Stirling. De acuerdo al resultado:

1. Si el valor está por debajo de 1 , la MEN es muy concentrada y se encuentra dependiente de una sola fuente primaria. Un sistema tal es muy dependiente y vulnerable a cualquier limitación en el suministro de su mayor fuente de generación. 
2. Si el valor es mayor que 2 , se considera un sistema diverso, es decir que utiliza varias y distintas fuentes primarias sin que uno sea predominante; por lo tanto puede ser considerado seguro.

TABLA 3.

Calculo del indicador Stirling.

\begin{tabular}{|l|l|l|l|}
\hline $\begin{array}{l}\text { Combinatoria } \\
\text { (pi x pj) }\end{array}$ & Resultado & $\begin{array}{l}\text { Valor de } \\
\text { disparidad }\end{array}$ & $\begin{array}{l}\sum \text { dij x pi x } \\
\text { pj }\end{array}$ \\
\hline TH & 0.07820371 & 2 & 0.15640742 \\
\hline TG & 0.06717828 & 4 & 0.26871313 \\
\hline TE & 0.07078431 & 6 & 0.42470583 \\
\hline TB & 0.04384585 & 8 & 0.35076681 \\
\hline HG & 0.01301731 & 2 & 0.02603463 \\
\hline HE & 0.01371606 & 4 & 0.05486425 \\
\hline HB & 0.00849613 & 6 & 0.05097676 \\
\hline GE & 0.01178232 & 2 & 0.02356465 \\
\hline GB & 0.00729831 & 4 & 0.02919325 \\
\hline EB & 0.00769008 & 2 & 0.01538015 \\
\hline $\begin{array}{l}\text { Indice de } \\
\text { diversidad } \\
\text { de Stirling al } \\
\text { 2014 }\end{array}$ & & & \\
\hline
\end{tabular}

Tabla 3.

El valor máximo del indicador para 5 fuentes es de 1,60. Este valor se obtiene suponiendo que las plantas de generación participantes en la MEN, se encuentra en un balance perfecto $(20 \% \mathrm{C} / \mathrm{U})$, se realiza el mismo proceso anterior y se obtiene un valor máximo de 1,60. Esto muestra que no es posible pretender alcanzar un valor mayor a 2 debido a que no hay variedad, pues el valor de referencia 2 es para un sistema con al menos 8 fuentes de generación distintas principalmente. EL indicador Stirling (IS), aplicado a la MEN 2013 muestra un resultado de1.4.

Este resultado muestra el grado de diversidad que Nicaragua posee, donde no es posible alcanzar un valor aproximado a 2, mostrando que la oferta energética se encuentra concentrada y dependiente de una sola fuente primaria para la generación de energía eléctrica y vulnerable a cualquier limitación del energético más utilizado en la generación de energía en Nicaragua. La Matriz energética de Nicaragua (MEN) se encuentra concentrada en plantas térmicas, plantas que utilizan el recurso energético petróleo, que tiene un alto costo y es un energético importado.

Finalmente el Indicador Stirlin propone evaluar concentrándose en la diversidad de la MEN, pero desagregando este el IS en sus tres componentes básicas, podemos concluir que nuestro sistema posee variedad 5/8 (5 fuentes primarias de 8 fuentes existentes principalmente) razón por la que Nicaragua no puede acercarse a un grado de diversidad mayor de 2 por poseer únicamente el 62,5\% (convirtiendo la fracción en porcentaje)de diversidad ; disparidad (cada una de las fuentes es distinta de la otra en cuanto a recursos energéticos, es decir ninguna depende del mismo energético) es lo que posee en un $100 \%$, es decir no se relacionan en cuanto al uso de energéticos.

El equilibrio, es lo que afecta más el grado de diversidad, puesto que el 54\% de la MEN utiliza el energético petróleo, razón por la que el balance o equilibrio, hace ver al portafolio energético como un mercado muy concentrado aun cuando se ha reducido considerablemente la generación térmica, (en relación porcentual con las otras fuentes primarias de generación) en relación a los años anteriores.

Indicador margen de reserva - (RM)

Este es in indicador (grafico 3) determinista e indica el residuo de energía luego de haber cubierto la demanda de todo un año; es el excedente que indica en el corto plazo seguridad del abastecimiento de 
energía eléctrica para los bloques de consumo en que se divide la demanda de energía eléctrica en Nicaragua. El resultado del indicador brinda la información necesaria para suponer el cierto grado de seguridad que presento la MEN en años anteriores. El margen de reserva se calcula en base a la capacidad instalada total en la MEN menos la demanda máxima de potencia de cada año. Dicha información se encuentra disponible en los portales web de instituciones del estado de Nicaragua como el INE.

TABLA 4.

Margen de reserva del Sistema interconectado nicaragüense 2005 al 2014.

\begin{tabular}{|l|l|l|l|l|l|l|l|l|l|l|l|}
\hline \multicolumn{2}{|c|}{ Margen de reserva del Sistema interconectado Nicaragüense 2005 al 2014 } \\
\hline & 2005 & 2006 & 2007 & 2008 & 2009 & 2010 & 2011 & 2012 & 2013 & 2014 \\
\hline $\begin{array}{l}\text { Capacidad } \\
\text { instalada } \\
\text { total en la } \\
\text { MEN }\end{array}$ & 752.6 & 746.2 & 813.5 & 874.7 & 969 & $1,060.10$ & $1,093.70$ & $1,267.70$ & $1,272.10$ & $1,313.08$ \\
\hline $\begin{array}{l}\text { Demanda } \\
\text { máxima } \\
\text { de } \\
\text { potencia }\end{array}$ & 482.8 & 500.8 & 507.4 & 506.27 & 524.5 & 538.9 & 569.5 & 609.9 & 620.1 & 636.1 \\
\hline $\begin{array}{l}\text { Margen } \\
\text { de } \\
\text { reserva }\end{array}$ & 269.8 & 245.4 & 306.1 & 368.43 & 444.5 & 521.2 & 524.2 & 657.8 & 652 & 676.98 \\
\hline
\end{tabular}

Tabla 4.

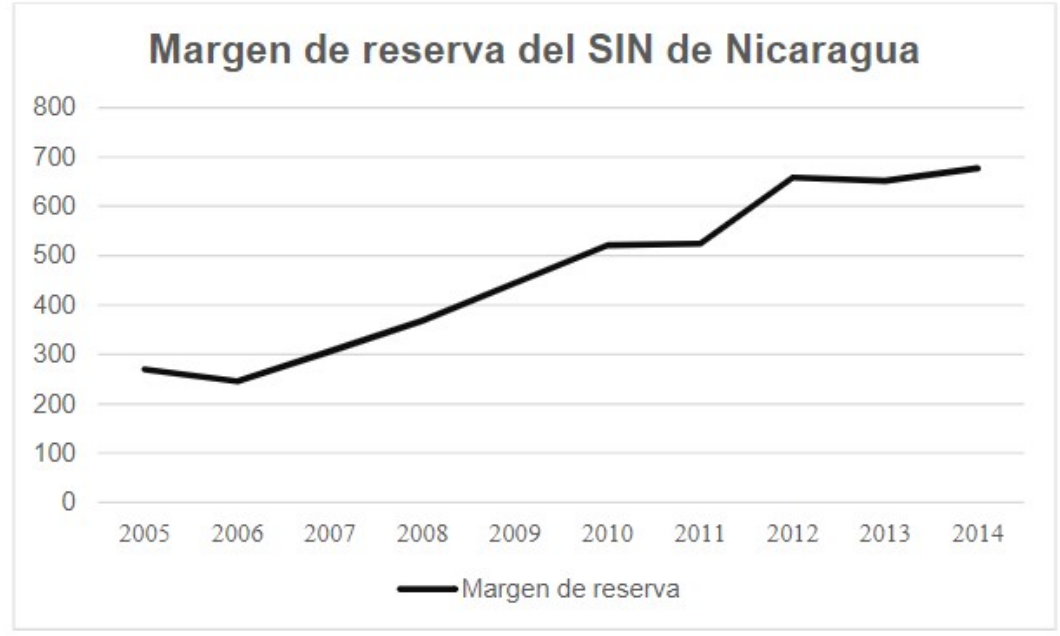

GRAFICO 3.

Margen de reserva del Sistema interconectado nicaragüense 2005 al 2014 en MW. Grafico 3.

El grafico 3 muestra el superávit de potencia de la MEN, se observa que Nicaragua desde años anteriores ha tenido un sistema con sobrecapacidad instalada. Pero esto no brinda información acerca de la operación del sistema sino que muestra que el sistema estaba en capacidad de cubrir la demanda con la potencia instalada efectiva, situación que presenta claramente las limitaciones de este indicador, que no toma en cuenta la operación del sistema sino que únicamente se centra en la capacidad total disponible, sin considerar incidentes negativos que afecten la disponibilidad de unidades o plantas de generación de energía eléctrica enteras. Limitaciones como reservas de los embalses, tamaños de plantas, distribución de la cartera energética (diversidad), tecnología y tasa de fallos.

Indicador pérdida del mayor generador - (LU) 
A diferencia del indicador RM, este indicador de SSE evalúa la situación de la MEN, al momento que no se pueda contar con la planta generadora de mayor capacidad por cualquiera que sea la naturaleza de la indisponibilidad. El indicador es obtenido de la relación entre el indicador RM y la cantidad de potencia de planta de mayor capacidad instalada. La planta de mayor capacidad es la Planta GEOSA con una capacidad de $106 \mathrm{MW}$ y como el margen de reserva se mantiene en el periodo de estudio mayor a los 269 MW significa que el valor de este indicador siempre es mayor a la unidad.

Si LU > 1, entonces podemos perder la unidad de mayor tamaño, sin afectar la continuidad del servicio asegurando el suministro a los consumidores finales al momento de las contingencias.

Índice de concentración de mercado - (ICM)

Este es un índice con amplia gama de aplicaciones a las ciencias y la ingeniería, y en este caso será aplicado para medir la competitividad de las fuentes primarias de generación de energía eléctrica presentes en el mercado nacional, es decir como se ha comportado el mercado eléctrico nacional en el periodo de estudio. Para el calcular el ICM, es necesario contar con el porcentaje de participación de cada uno de los tipos de plantas presentes en la MEN, desde el año 2000 hasta el 2014, luego los porcentajes se multiplican para encontrar el valor del ICM.

SIN

Concentracion de mercado de SIN

Concentracion de mercado de SIN

\begin{tabular}{|l|l|l|l|l|l|l|l|l|l|l|}
\hline Planta & 2005 & 2006 & 2007 & 2008 & 2009 & 2010 & 2011 & 2012 & 2013 & 2014 \\
\hline Termica & 68.61 & 73.14 & 74.28 & 67 & 72.4 & 65.7 & 68.9 & 60.26 & 50.28 & 48.63 \\
\hline Hidroelectrica & 15.36 & 10.44 & 10.35 & 17.18 & 9.21 & 14.84 & 12.41 & 11.24 & 11.85 & 9.64 \\
\hline Geotermica & 8.69 & 9.65 & 7.27 & 9.4 & 8.34 & 7.97 & 6.84 & 12.9 & 16 & 14.57 \\
\hline Eolica & 1 & 1 & 1 & 1 & 3.47 & 4.77 & 5.85 & 8.84 & 14.62 & 20.58 \\
\hline Biomasa & 7.33 & 6.77 & 8.1 & 6.4 & 6.54 & 6.68 & 5.96 & 6.76 & 7.25 & 6.55 \\
\hline ICM & 67128 & 49885 & 45272 & 69248 & 126204 & 247601 & 203915 & 522137 & 1010460 & 920720 \\
\hline
\end{tabular}

SIN

EL ICM (grafico 4) para Nicaragua posee un rango mayor al de 2000 (Competencia perfecta y balanceada), valor que se alcanza cuando los participación porcentual de las 5 fuentes de generación de energía eléctrica es igual para todas (20\%), cuando el valor se acerca a los 10000, se considera un mercado sin competencia justa en la que se encuentra un control casi monopolizado del mercado. En caso de que el valor sea de 10000, como en el caso de Nicaragua la competencia justa no existe y el mercado es controlado únicamente por una sola empresa que monopoliza el servicio, estanca la innovación, y contrala los precios a conveniencia. 


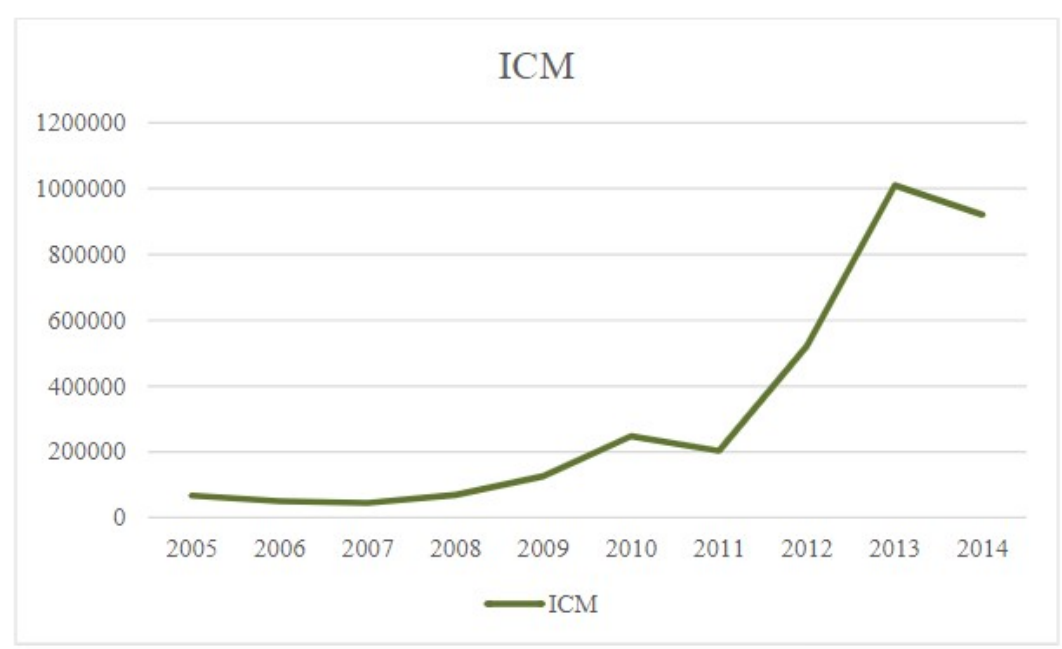

GRAFICO 4.

Índice de concentración de mercado

Grafico 4.

Como el grafico 4 muestra a lo largo de los años anteriores a 2014, se observa que el mercado eléctrico nacional (ME) se encontraba altamente concentrado, lo único que diferenció al ME de encontrarse monopolizado en esos años, fue que ya existían otros agentes generadores aunque con una baja participación en la MEN. A inicios del periodo de estudio en el año 2000-2001, se encontraba altamente concentrado con generación térmica, años en los que la participación al ME de la generación térmica alcanzo hasta un $70 \%$, luego se introdujeron nuevos proyectos de generación de biomasa y proyectos geotérmicos, que contribuyeron a la reducción del ICM.

El grafico 4 muestra una constante variación en el valor del índice, porque por 9 años se mantuvo la MEN con 4 tipos de generación, estos supone un crecimiento vertical del mercado eléctrico en donde no se aumentó el tipo de fuente primaria para la generación de energía, sino que solo se creció en capacidad instalada, en 2009, se introduce la primera planta eólica, (Amayo) lo que causo un impacto al mercado, el que experimento un crecimiento vertical y horizontal, al contar a ese momento con 5 fuentes primarias distintas para la generación de energía eléctrica.

Cuando el mercado está concentrado no es competitivo y eso sucedió en años anteriores y sucede actualmente, ¿̨por qué se afirma esto? Porque el cálculo del precio del servicio Energía Eléctrica se realiza en base a las proyecciones del precio internacional del petróleo para que de esta manera se genere ganancia a la distribuidora aun cuando el precio internacional del petróleo suba, en caso contrario (baje el costo) el valor calculado tiene un desvió que será compensado en los siguientes cálculos, y así sucesivamente.

Lo dicho anteriormente evidencia la dependencia de una sola empresa (fuente primaria de energía), en el cálculo de precios. Además de las afectaciones a la SSE que conlleva el poseer un mercado concentrado en el que la falta de competencia genera estancamiento en la innovación, mayor eficiencia y calidad del servicio, y asequibilidad del servicio por el simple hecho de poseer un mercado competitivo.

Evaluación de la productividad del uso de energéticos en la generación de energía eléctrica

Se realizó la evaluación de la productividad del empleo de recursos energéticos del tipo térmicos derivados del petróleo, hidroeléctrico, geotérmico y de biomasa y los resultados se muestran en la siguiente tabla. 
Revista Iberoamericana de Bioeconomía y Cambio Climático, 2015, vol. 1, núm. 2, Agosto-Diciembre,...

EVALUACIÓN DE LA PRODUCTIVIDAD DEL EMPLEO DE RECURSOS ENERGÉTICOS DEL TIPO TÉRMICOS DERIVADOS DEL PETRÓLEO, HIDROELÉCTRICO, GEOTÉRMICO Y DE BIOMASA

Promedio de ritmo de crecimiento de la productividad de los factores, la eficiencia tecnicay la tecnologia del uso de recursos energeticos para genrear energia electrica en Nicaragua

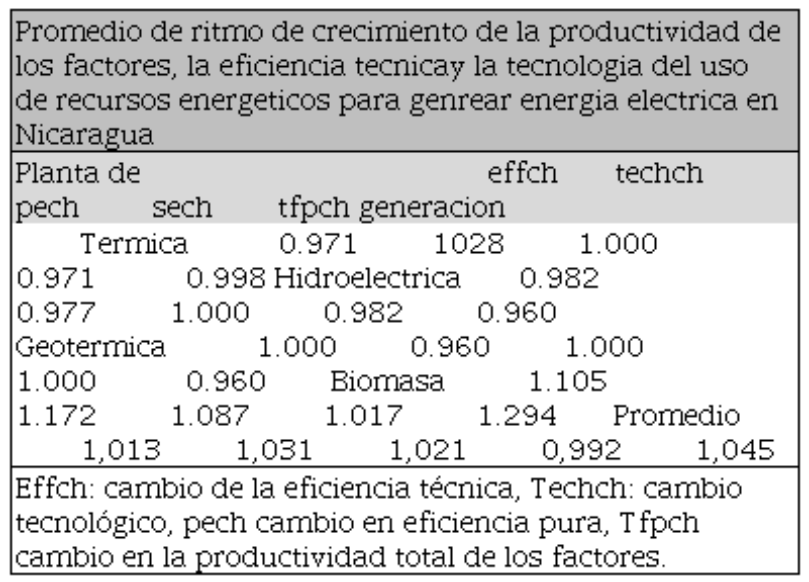

Promedio de ritmo de crecimiento

De los resultados se obtiene que en promedio el ritmo de crecimiento interanual de la productividad total de los factores, durante el periodo de estudio para los recursos estudiados fue de $4.5 \%$ eficiente. La productividad total del uso de los recursos se explica fundamentalmente por el ritmo de crecimiento interanual de la eficiencia técnica que resultó con un $1.3 \%$ y un 3.1\% de cambio tecnológico, a su vez podemos valorar que esta eficiencia se explica por el ritmo de crecimiento de la eficiencia pura de $2.1 \%$ indicando que la capacidad y la asistencia técnica de los trabajadores en el proceso de generación de energía, y de igual manera se nota una desmejora en la economía de escala de $0.8 \%$.

El recurso con mayor índice de crecimiento interanual de productividad total de los factores fue la biomasa un índice de 1.294 es decir, un $29.3 \%$ de aumento de productividad en ritmo de crecimiento interanual, que es debido al cambio en la eficiencia técnica (10.5\%) interanual y un $17.2 \%$ de crecimiento en el cambio tecnológico que se explica con un crecimiento del $2.1 \%$ en la eficiencia pura y un 1.7 en la eficiencia de escala.

\section{CONCLUSIONES}

El indicador Stirling muestra que la Matriz energética de Nicaragua (MEN) se encuentra concentrada en plantas térmicas, plantas que utilizan el recurso energético petróleo, que tiene un alto costo y es un energético importado.

El indicador de margen de reserva muestra el superávit de potencia de reserva de la MEN, dado que Nicaragua desde años anteriores ha tenido un sistema con sobrecapacidad instalada.

El indicador de pérdida del mayor generador indica que debido al margen de reserva que se mantiene en el periodo de estudio podemos perder la unidad de mayor tamaño, sin afectar la continuidad del servicio asegurando el suministro a los consumidores finales al momento de las contingencias.

El índice de concentración de mercado muestra que la competencia justa no existe y el mercado es controlado únicamente por una sola empresa que monopoliza el servicio.

En la evaluación de la productividad del empleo de recursos energéticos del tipo térmicos derivados del petróleo, hidroeléctrico, geotérmico y de biomasa se obtuvo que el recurso con mayor índice de crecimiento interanual de productividad total de los factores fue la biomasa. 


\section{Referencias Bibliográficas}

Banco mundial (2015). Crecimiento demográfico (\% anual). http://datos.bancomundial.org/indicador/SP.POP.GR OW

Blanco-Orozco, Napoléon Vicente, Zúniga-González, Carlos Alberto, Arce, Eduardo, Jaramillo-Villanueva, José Luis, Solano, Luis Losilla, Colón García, Adelfa Patricia (2014). Evaluación integral Financiera, económica, social, ambiental y productividad del uso de bagazo de caña en la generación de energía Eléctrica en Nicaragua en ingenios no conectados a la red de energía eléctrica. Universitas (León) Vol. 5 (2) 2014, pag 42-51.

CIEMAT.2012. Situación de la energía en el mundo, Europa y España. http://www.energiasrenovables.ciemat.es/es peciales/energia/index.htm

Coelli, T.J., (1996). A guide to DEAP Version 2.1: A Data Envelopment Analysis (Computer) Program. Center for Efficiency and Productivity Analysis (CEPA) working paper, department of Econometrics. University of New England Armidale NSW 2351 Australia.http:www.une. edu.au/ econometrics/cepawp .htm IS S N 1327-435X, ISBN 1863894969.

Ferro, G y Romero, C. (2011). Comparación de medidas de cambio de productividad. Las aproximaciones de Malmquist y Luenberger en una aplicación al mercado de seguros. HAL Id: hal-00597946

Fergal, L., Morgan B., Howley, M., y Gallachóir, B. (2007). Security of Supply in Ireland 2007. Sustainable Energy Ireland (SEI). Energy Policy Statistical Support Unit. Ireland. Obtenido desde: http://www.seai.ie/Publications/Statistics_Publications/Energy_Security_in_I reland/ SEI_EPSSU_Security_of_Supply_Third_Report.pdf

J. Flores, J, Herrera-Toscano, J y Flores, S. (2014). Cambios en la productividad y sus determinantes en explotaciones lecheras de Cuba. Información técnica económica agraria: revista de la Asociación Interprofesional para el Desarrollo Agrario (AIDA), ISSN 1699-6887, №. 2, . págs. 187-207. Obtenido desde. http://www.aidaitea.org/aida-itea/files/itea/revistas/2014/110-2/(187- 207)\%20E18427.pdf

Molina, J. D., Martínez, V., \& Rudnick, H. (2009). Indicadores de Seguridad Energética: Aplicación al Sector Energético de Chile. In 2nd Latin American Meeting on Energy Economics (p. 22).

Ministerio de Energía y Minas. (2013). Parque de generación por tipo de fuente

Pedraja, F y Jiménez, J.(1994). La restricción de las ponderaciones en el análisis envolvente de datos: una fórmula para mejorar la evaluación de la eficiencia. Investigaciones económicas, 23(2).365-380.Obtenido desde: http:// www.fundacionsepi.es/revistas/paperArchive/May1994/v18i2a6.pdf

Quindós M., Rubiera F., Vicente M. (2003). “Análisis envolvente de datos: Una aplicación al sector de los servicios avanzados a las empresas del principado de Asturias”. Asociación española de profesores universitarios de matemáticas para la economía y la empresa. XI Jornadas Oviedo, 11 y 12 de Septiembre 2003 España. Disponible: http://www.uv.es/asepuma/XI/21.pdf

Retamales, G. (2005). Indicadores de Seguridad de Suministro Eléctrico (SSE) en Chile. Tesis de grado. Facultad de Ciencias Físicas y Matemáticas. Chile. Universidad de Chile.Scheepers, M.J.J., A.J. Seebregts, J.J. de Jong, J.M. Maters. (2007). EU Standards for Energy Security of Supply. Obtenido desde : http://www.ecn.nl/docs/libra ry/report/2007/e07004.pdf

Schuschny, A. (2007). El método DEA y su aplicación al estudio del sector energético y las emisiones de CO2 en América Latina y el Caribe. ISSN electrónico 1680-8789. LC/L.2657-P. Obtenido desde: http://repositorio.c epal.org/bitstream/handle/11362/4752/S0700014_es.pdf?

Stirling, A. (2003). On the Economics and Analysis of Diversity, Electronic Working, Papers Series.28. Obtenido desde: http://www.sussex.ac.uk/Units/spru/publications/imprint/sewps/sewp28/sew p28.pdf

Ventosa, A. (2010). Modelos de Sistemas de Energía Eléctrica: Índices, Medidas y Criterios de Fiabilidad. Instituto de Postgrado y Formación Continua. Universidad Pontificia de Madrid. España. 